\title{
AÇÕES DESENVOLVIDAS JUNTO AOS FAMILIARES DE PESSOAS COM COVID-19: RELATO DE EXPERIÊNCIA
}

Fabíola Moreira Casimiro de Oliveira'

ORCID: 0000-0003-2983-6621

Ailma de Souza Barbosa"

ORCID: 0000-0001-7418-5771

Lenilma Bento de Araújo Meneses"I ORCID: 0000-0001-7966-7411

Verônica Ebrahim Queirogalv

ORCID: 0000-0001-8816-5851

Maria do Socorro Sousa da Silvav ORCID: 0000-0002-7931-834X

Ana Lúcia Teixeira de Limavı ORCID: 0000-0003-1761-3316

I, III IV, V Prefeitura Municipal de João Pessoa. João Pessoa, Paraíba, Brasil.

III, VI Universidade Federal da Paraíba. João Pessoa, Paraíba, Brasil.

IIII,IV,V,VIMembros do Departamento Científico de Enfermagem na Atenção Básica.

Autor Correspondente: Fabíola Moreira Casimiro de Oliveira E-mail: fabiolamco@gmail.com

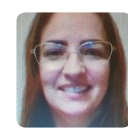

Como citar:

Oliveira FMC, Barbosa AS, Meneses LBA, Queiroga VE, Silva MSS, Lima ALT. Ações desenvolvidas junto aos familiares de pessoas com coronavírus: relato de experiência. In: Teodósio SSS, Leandro SS

(Orgs.). Enfermagem na atenção básica no contexto da COVID-19. 2.ed.rev. Brasília, DF : Editora ABEn, 2020. p. 55-60. (Série Enfermagem e Pandemias, 3). https://doi.org/10.51234/aben.20.e03.c08

\section{INTRODUÇÃO}

A COVID-19, doença causada pelo novo coronavírus, de transmissão rápida, foi se espalhando para outros Continentes, chegando de forma avassaladora a quase todos os países, e tem exigido que os serviços de saúde de todo o mundo se reorganizem para atenderem, de forma resolutiva, as necessidades de saúde da população ${ }^{(1,2)}$.

A situação de pandemia, em nosso país, trouxe algumas revelações. Por um lado, que o Sistema Único de Saúde (SUS) é o melhor e maior sistema do mundo; e por outro, que é necessária uma melhor estrutura física, organizacional, de gestão e de equipamentos, para que as Redes de Atenção em Saúde (RAS) consigam manter suas atividades de forma integradas e resolutivas. Apenas por meio da organização dos níveis de atenção, são definidos os papéis e fluxos para o atendimento dos sintomáticos respiratórios, incluindo a COVID-19, e de todos os usuários que apresentem as mais diversas necessidades de saúde, evidenciando o alcance da integralidade do cuidado, perpassando pelo trabalho imperativo dos Agentes Comunitários de Saúde (ACS), da equipe da Atenção Primária de Saúde (APS), seguindo até $\mathrm{o}$ atendimento em unidade hospitalares de média e alta complexidade, incluindo as Unidades de Terapias Intensivas (UTI) ${ }^{(3)}$.

A APS revela-se fundamental como porta de entrada do SUS no combate à pandemia, oferecendo atendimento aos casos leves, e identificação precoce dos casos graves, que necessitam ser encaminhados para os serviços especializados, respeitando o fluxo do cuidado em todos os níveis da atenção em saúde ${ }^{(4)}$.

Além dos princípios gerais da APS, a Estratégia de Saúde da Família (ESF) tem caráter substitutivo em relação à RAS, nos territórios de atuação das Equipes de Saúde da Família, por atuar no território adscrito; realizar cadastramento domiciliar, diagnóstico situacional; desenvolver ações dirigidas aos problemas de saúde de maneira pactuada com a comunidade 
onde atua; cuidar dos indivíduos e das famílias ao longo do tempo, frente aos problemas de saúde-doença da população de sua responsabilidade; desenvolver atividades, de acordo com o planejamento e a programação, realizados com base no diagnóstico situacional, e tendo, como foco, a família e a comunidade ${ }^{(5)}$. Todo esse conhecimento e estrutura em Redes colocam a APS como a principal aliada ao enfrentamento do Cononavírus.

A atenção integral à saúde de toda a família é um objetivo inalienável do SUS, que se fundamenta nos princípios da universalidade, integralidade e equidade. A partir desses valores, deve garantir a todo cidadão e cidadã uma atenção integral diante das suas necessidades de saúde, possibilitando o acesso qualificado e a assistência humanizada, sem preconceitos, discriminação ou privilégios ${ }^{(6)}$.

A ESF emerge como um modelo reorganizador do SUS e privilegia o núcleo familiar como instituição necessária para intervir no processo saúde-doença, nas ações de promoção da saúde, prevenção de doenças e agravos, reabilitação e vigilância em saúde. ${ }^{6}$

No enfrentamento da COVID-19, a Atenção Primária em Saúde, por meio da ESF, tem exercido papel central na prevenção e controle de doenças, sendo capaz de colaborar estrategicamente com a redução do risco de transmissão da doença, a partir do diagnóstico precoce, acompanhamento e monitoramento do indivíduo, família e comunidade ${ }^{(7)}$. Assume-se, portanto, papel resolutivo frente aos casos leves e de identificação precoce e encaminhamento efetivo e correto dos casos graves, mantendo a coordenação do cuidado desses últimos.

A estratificação de intensidade da Síndrome Gripal (SG) é a ferramenta primordial para definir a conduta correta para cada caso, seja para manter o paciente na APS/ESF, ou para encaminhá-lo aos centros de referência, urgência/emergência ou hospitais ${ }^{(8)}$. Os casos de SG, sem complicações ou sem comorbidades de risco, serão conduzidos pela APS/ESF. Logo, faz-se obrigatório o acompanhamento desses usuários pelos profissionais ao longo do curso da doença, utilizando estratégias coletivas, interprofissionais e intersetoriais ${ }^{(8)}$.

Sensibilizadas por essa conjuntura, a equipe de saúde buscou estratégias de enfrentamento à COVID-19, de forma a desenvolver ações junto aos familiares de pessoas com COVID 19, para conter a disseminação no território assistido. Desse modo, deve-se evidenciar o Sistema Único de Saúde e Atenção Primária à Saúde forte.

\section{OBJETIVOS}

Descrever as ações desenvolvidas junto aos familiares de pessoas com COVID 19, realizadas por uma Equipe de Saúde da Família do município de João Pessoa.

\section{METODOLOGIA}

Trata-se de um estudo descritivo, como relato de experiência, a partir dos recursos técnicos da abordagem qualitativa, segundo Minayo ${ }^{(9)}$, de acordo com a concepção pedagógica, centrada no sujeito de aprendizagem, focada no desenvolvimento de competências, e a consequente valorização da experiência, para que ocorra aprendizagem significativa.

O cenário, em análise, foi uma Unidade Saúde da Família (USF), pertencente ao Distrito Sanitário V, do município de João Pessoa/Paraíba (PB). Esta USF atua em um prédio próprio, com uma Equipe de Saúde da Família, composta por 20 trabalhadores: um médico, uma enfermeira, uma dentista, uma técnica de enfermagem, uma auxiliar de saúde bucal, dez agentes comunitários de saúde, uma farmacêutica, uma auxiliar de farmácia, uma auxiliar administrativa, uma auxiliar de serviços gerais, um vigilante e uma gerente do Núcleo de Apoio à Saúde da Família (NASF). Nesse novo contexto de pandemia, muitos profissionais se afastaram do serviço por condições de doença pré-existentes e adquiridas, ficando uma quantidade mínima de profissionais: em torno de dez pessoas na atenção do cuidado aos usuários.

O público alvo foram os familiares de pessoas acometidas pelo coronavírus na área adscrita do serviço de saúde, tomando, por base, a realização da testagem rápida, na própria Unidade de Saúde, conforme 
critérios estabelecidos no protocolo municipal de agendamento, a partir do $10^{\circ}$ dia do início dos sintomas de SG, com risco para Síndrome Respiratória Aguda Grave (SRAG), coronavírus \COVID-19. Outros sintomas se apresentaram como febre, tosse seca, conjuntivite, dor de garganta, cefaleia, diarreia, perda do olfato ou paladar, principalmente desconforto respiratório e falta de ar. O laudo conclusivo seria entregue ao usuário, e consolidados os números positivos e negativos em planilha exclusiva de testagem, impressos padronizados pela gestão municipal.

A articulação com a rede de saúde também é fundamental, incluindo Serviço Móvel de Urgência (SAMU), Unidades de Pronto Atendimento (UPAS) e Hospitais de Referência, com impresso elaborado especialmente para casos suspeitos elou confirmados de COVID-19.

Além do usuário, os familiares precisavam ser orientados quanto ao cuidado com o doente em domicílio, atendendo ao isolamento domiciliar e medidas protetivas dos familiares dentro da casa, por meio da criação de instrumentos educacionais e orientações de saúde, como: jingle (letra e música); criação de vídeos com os trabalhadores, professores e estudantes com orientações de etiqueta de higiene; esclarecimento sobre cuidados e formas de contágio da doença e criação de Podcast.

\section{RESULTADOS}

A Saúde Pública foi surpreendida, no mês de março, com um contexto novo de Pandemia do novo coronavírus. Como repensar o processo de trabalho da equipe no momento em que a única medida de conter a disseminação do vírus era ficar em casa? Neste tempo de distanciamento social, a equipe não pôde realizar visitas domiciliares de rotina, e os ACS foram afastados dos seus territórios. Que estratégias utilizar para acompanhar e aproximar esses usuários do território aos profissionais da equipe? Como fazer a APS manter suas atividades e criar fluxo de atendimento para os sintomáticos respiratórios?Pensar coletivamente, problematizar e refletir o processo de trabalho e o cuidado ofertado foi uma estratégia precípua. Tudo começou em meados de abril, ainda no período da imunização, com a oferta de vacina contra influenza dos acamados, idosos e restritos ao lar. Esse trabalho realizado pela equipe de enfermagem, casa a casa, já foi primordial. Ao indagar como andava a saúde, recebíamos como resposta que, na casa, existiam pessoas com febre, tosse e dor de garganta, sintomas mais frequentes da atual doença; e a mídia já noticiava casos confirmados no município de João Pessoa com vítimas fatais. No próprio domicílio, as orientações eram dadas, alertando para os sinais de agravo e com recomendação da necessidade de distanciamento social e de orientações e reforço de medidas de higiene ao usuário acometido e seus familiares.

Como estratégia de enfrentamento à COVID-19, os profissionais de saúde foram capacitados pelo Ministério da Saúde e a gestão municipal viabilizou os protocolos de manejo clínico, assim como propiciou medidas de biossegurança para garantir a proteção dos trabalhadores e usuários. Nesse ínterim, os fluxos de atendimentos na Unidade de Saúde foram repensados, utilizaram-se da gestão da clínica para organizar os atendimentos de demandas dos sintomáticos gripais e de sua família, como também das outras demandas da Atenção Primária.

A USF disponibilizou uma Farmácia Polo que permaneceu aberta, e garantiu a dispensação dos medicamentos de uso regular da APS, com fluxo orientado e com controle de higienização.

Os profissionais tiveram que se reinventar, vencer desafios e buscar ferramentas tecnológicas para melhorar a comunicação e o diálogo com a comunidade a fim de realizar o monitoramento à distância. Sendo assim, utilizou-se do teleatendimento aos usuários de risco e os atendimentos presenciais para os não riscos. Foi um momento bem difícil, pois muitas incertezas, medo e tensão tomavam conta dos profissionais e da comunidade.

Os atendimentos às linhas de cuidado permaneceram com agenda programada (saúde da mulher, saúde da criança, saúde do idoso, saúde mental, saúde do trabalhador), embora com redução de agenda, sempre com hora marcada e seguindo com todos os protocolos preconizados. A Saúde Bucal, embora com exposição maior de risco de contaminação, permaneceu com atendimentos de urgências e, também, colaborando com o trabalho da equipe. 
Inicialmente, por não ter nenhum caso confirmado no território, os usuários foram resistentes em seguir as orientações do uso da máscara e em ficar em casa. Os dias foram se passando, os testes rápidos chegaram e os positivos se confirmaram. Foi, nesse momento, que a equipe percebeu como é difícil cumprir protocolos quando os determinantes e condicionantes da saúde, como alimentação e moradia, não favorecem para a população mais vulnerável. Surgiram os questionamentos: Como isolar, em um único cômodo, uma única pessoa quando a casa tem cinco ou seis moradores? Como fazer para criar uma rede de proteção quando não é fácil deixar de se abraçar? Como incorporar o uso da máscara na vida das pessoas? Como ajudar os familiares das pessoas com COVID 19?

Foram inquietações que provocaram sentimento de impotência, advindo da implicação do profissional ao seu território de atuação, da importância do vínculo e do reconhecimento do território, no sentindo de conhecer o contexto em que vivem as famílias. E por meio do diálogo, identificar recursos básicos, como água e sabão; respeitar as limitações da ambiência; fazer adaptações no domicílio, como armar redes na sala, nos corredores entre os quartos; separar os utensílios pessoais; e manter contato, através de aplicativos de mensagens, com a equipe de saúde. Estas foram algumas ações/estratégias desenvolvidas. Ressalta-se, ainda, a importância da tecnologia de informação, neste momento de Pandemia, que tem aproximado as pessoas e facilitado a comunicação.

Sabe-se que o trabalho na APS acontece de forma multiprofissional e, neste contexto, constata-se a grande importância dos profissionais de nível médio (técnico de enfermagem, auxiliar de saúde bucal, recepcionistas, auxiliar administrativo e de serviços gerais, gerente e agente comunitário de saúde) dessa equipe, realizando, junto com os cirurgiões dentistas e farmacêuticos, práticas interprofissionais e colaborativas.

Portanto, a força do SUS está na base, no fazer, no ser, no viver, no dia a dia, por isso tem mostrado um trabalho fantástico e resolutivo, na medida em que mostra, para todos, que é possível se reinventar, mostrar por que somos fortes, e essa força parte da vontade e do desejo de transformar as práticas de cuidado na perspectiva da necessidade do usuário.

Cuidar das pessoas e enfrentar o adoecimento talvez tenham sido os maiores desafios para os profissionais da equipe, quando quatro trabalhadores foram infectados pelo vírus e tiveram que enfrentar o medo e a tensão de não saberem como seria esse percurso. Outro momento difícil foi o óbito de um usuário muito querido pela equipe e pela comunidade que, apesar de morar sozinho, os familiares estão sentindo sua ausência, principalmente a ex companheira que, todos os dias, tem procurado a unidade de saúde, sempre muito queixosa, que é acolhida com dedicação, escuta qualificada e amorosa.

Outra forma de oferta de cuidado tem sido as Práticas Integrativas e Complementares (PIC's), como a auriculoterapia e os florais, que têm contribuído para melhorar a saúde mental e emocional dos trabalhadores e dos usuários.

Essa experiência trouxe resultados positivos, como a criação de fluxos de atendimentos, com redução do número de cadeiras na recepção e consultórios, favorecendo o distanciamento de, no mínimo, 1 (um) metro, bem como sinalização horizontal ou vertical de direções para área específica de atendimento aos sintomáticos respiratórios, e outra para não sintomáticos; uso obrigatório de máscara de proteção facial para usuários e trabalhadores e reforço da lavagem das mãos e borrifação de álcool líquido a 70\%, na entrada da unidade para todos; organização das demandas de atendimentos dos grupos prioritários como gestantes, puérperas/ recém-nascidos, portadores de doenças crônicas degenerativas, entre outros.

A gestão municipal disponibilizou um impresso para registro do atendimento específico ao sintomático respiratório e quadro de monitoramento a cada 48 horas, por telefone; e o preenchimento manual ou online da ficha de notificação compulsória obrigatória e imediata até 24 horas para informação de boletim à Vigilância Epidemiológica (VIEP) de casos suspeitos ou confirmados foi intensificado. Isso potencializa as tomadas de decisões de medidas restritivas para o combate à disseminação da epidemia, pela equipe na área adscrita. Também os dados se mantêm atualizados e os profissionais têm ciência da realidade de seu território. $\mathrm{A}$ parceria com a Gerente da unidade, com a equipe do Distrito Sanitário fortalece o trabalho em equipe. 
A divulgação e repercussão da música no território adscrito e áreas adjacentes, através do motosom do jingle e nas redes sociais, como também nas principais rádios do município de João Pessoa, foram muito importantes para aliviar o estresse diante de um momento tão tenso e receber uma informação acessível.

\section{CONTRIBUÍÇÕES PARA ENFERMAGEM}

Mais uma vez, a enfermagem se destaca pelo protagonismo, junto à equipe de saúde da família, aos usuários e à população da área adscrita. A organização de espaço, as estratégias realizadas e o estímulo ao trabalho, em equipe multiprofissional e interdisciplinar, proporcionaram um diferencial no enfrentamento e combate à COVID-19, sem esquecer as demandas inerentes da APS.

Para os profissionais, surgiu a necessidade de se reinventar no processo de trabalho, e a maneira de desenvolver ações estratégicas de promoção à saúde e prevenção da doença, em tempos de pandemia. A experiência vivenciada pelos trabalhadores, especialmente para a equipe de enfermagem, durante a vacinação para influenza, que ocorreu no início da pandemia para idosos acamados em domicílio, possibilitando identificar sintomáticos gripais e, no momento, já orientar sobre isolamento domiciliar diante as condições reais e, de forma prévia, ir preparando a comunidade sobre o atual contexto do problema de emergência de Saúde Pública.

À medida que a equipe otimizava a visita para a busca ativa de casos, evidenciava-se o caráter de humanização e de vínculo com os usuários acometidos pela doença, junto aos seus familiares, por meio do desenvolvimento de ações estratégicas voltadas ao enfrentamento da COVID-19, na área de abrangência da equipe.

Infere-se que essa iniciativa possa inspirar outros atores no planejamento e execução de ações estratégicas, e sirva de modelo para os demais profissionais, para repensarem suas práticas, a partir de um novo olhar, um novo fazer.

Acredita-se que este relato possa servir de base para promover o comparativo com novos estudos e relatos de experiências em muitos cenários, assim como possibilitar outras experiências e colaborar com a ciência.

\section{LIMITAÇÃO DO ESTUDO}

Pode-se inferir, como limitação, o pouco conhecimento dos profissionais do uso das tecnologias da informação, como ferramenta de aproximação, comunicação e orientações em saúde com a comunidade e como instrumento de vigilância em saúde. Assim como identificar, neste momento de distanciamento social, parcerias intersetoriais que possam acolher as demandas do território, e contribuir com o trabalho da equipe de saúde, em busca de superar os gargalos e silêncios ao despreparo dos profissionais pela integralidade do cuidado e pelo trabalho em equipe.

\section{CONDIDERAÇÕES FINAIS}

Ao refletir sobre o contexto mundial e local que esta Pandemia do novo coronavírus provocou no SUS, inicialmente, sobrevieram sentimentos de incertezas, inquietações, medo e tensão. Passados os primeiros impactos, desafiados por esta nova realidade e pelo desejo de construir formas de enfrentamento dos dinâmicos e complexos problemas de saúde da comunidade, os profissionais soltaram suas vozes e impulsionaram adaptações, e a ressignificação do cuidado ofertado aos usuários do SUS foi evidente, uma característica de uma APS forte.

As facetas desveladas nesta experiência vivenciada na APS/ESF, com o advento da Pandemia, lançaram rumos a outros caminhos desafiadores. A certeza de que a consolidação dos processos, disparados na singularidade de cada território da APS/ESF, deve perpassar pelo apoio institucional, pelo financiamento, pela valorização do trabalhador, pela efetivação da integração das equipes de saúde, pela prática colaborativa, pelo reforço das tecnologias de saúde e comunicação, como potencializador da vigilância em saúde e da gestão da clínica. 
Os resultados apresentaram-se favoráveis, por meio de estratégias viáveis e significativas, para o real entendimento da situação de saúde-doença da população, colaboração do usuário doente e de seus familiares, levando em consideração o contexto de vida, condições de moradia, social e sanitária, para a nova forma de cuidar da saúde de maneira individual e coletiva.

Por fim, tem-se a consciência de que o caminho a ser trilhado na busca do cuidado de qualidade é árduo, porém vislumbra-se um SUS que resiste, que está vivo, e temos o privilégio de fazer parte desta construção. Diante dessa premissa, não se pretendeu apresentar soluções prescritivas, pois se entende que, à medida que novas situações se apresentam, novos modos de pensar e agir também vão acontecendo na APS/ESF. Esse é o SUS que temos e queremos!!

\section{REFERÊNCIAS}

1. WHO. WHO announces COVID-19 outbreak a pandemic. 2020. [Acesso em 24/06/2020]. Disponível em: http://www.euro.who.int/en/health-topics/health-emergencies/coronavirus-covid19/news/news/2020/3/ who-announces-covid-19-outbreak-a-pandemic.

2. Gorbalenya AE, Baer SC, Baric RS. Et al. The species Severe acute respiratory syndrome-related coronavirus: classifying 2019-nCoV and naming it SARS-CoV-2. Nat Microbiol, p. 536-544. 2020. [Acesso em 24/06/2020]. Disponível em: www. ncbi.nlm.nih.gov/pmc/articles/PMC7095448/.

3. Brasil. Conselho Nacional de Secretários de Saúde-CONASS. Guia Orientador para o enfrentamento da pandemia Covid-19 na Rede de Atenção à Saúde. Brasília, maio de 2020.

4. Brasil. Ministério da Saúde. Protocolo de manejo clínico do Coronavírus (COVID-19) na Atenção Primária à Saúde -Versão 09-Brasília, abril 2020.

5. Brasil. Ministério da Saúde. O SUS de A a Z. Garantindo saúde nos municípios. Série F. Comunicação e Educação em Saúde. 3 ed. Brasília - DF. 2009

6. Ramalho L, Silva JA. Projeto Pedagógico (recurso eletrônico) - Natal: SEDIS-UFRN, 2017. [Acesso em 24/06/2020]. Disponível em: https://lais.huol.ufrn.br/wp-content/uploads/2020/06/Bibliografia-para-fase-2-projeto-pedagogicoEdital-029-2020.pdf.

7. Barbosa SP, Silva AVFGA. A Prática da Atenção Primária à Saúde no Combate da Covid-19. APS em Revista, v. 2, n. 1, p.17-19. 2020. [Acesso em 24/06/2020]. Disponível em: https://apsemrevista.org/aps/article/view/62/43.

8. Brasil. Ministério da Saúde. Protocolo de Manejo Clínico para o Novo Coronavírus (2019-nCoV). Brasília- DF, 2020:32p. [Acesso em 24/06/2020]. Disponível em: https://portalarquivos2.saude.gov.br/images/pdf/2020/fevereiro/11/protocolomanejo-coronavirus.pdf.

9. Minayo MCS. O desafio do conhecimento: pesquisa qualitativa em saúde. 13 ed. São Paulo: Hucitec, 2015. 406 p. 